\title{
Asymptomatic osteomyelitis of the symphysis pubis
}

\author{
T.W. Evans, R.S. Evely and S.K. Morcos \\ Department of Radiology, Northern General Hospital and Lodge Moor Hospital, Sheffield, UK.
}

\begin{abstract}
Summary: A patient presenting with pyrexia of unknown origin is described. Extensive radiological investigations over a six month period failed to localize an infective focus. Osteomyelitis of the symphysis pubis was found at post mortem. The clinical and radiological implications are discussed.
\end{abstract}

\section{Introduction}

Infection of the symphysis pubis is a rare condition accounting for less than $1 \%$ of cases of haematogenous osteomyelitis (McHenry et al., 1975). Pelvic surgery, trauma and drug abuse have been reported as predisposing causes. Pain and localized tenderness are the usual presenting complaints. (Busto et al., 1982).

We report a case of osteomyelitis of the symphysis pubis in a patient with no predisposing cause and no pain or local tenderness. Radiological investigations including isotope gallium scan failed to establish the diagnosis which was eventually suggested six months after the initial date of presentation.

\section{Case History}

A 70 year old woman was admitted with bilateral parotitis and septicaemia. Staphylococcus aureus was isolated from blood cultures, nasal and throat swabs. Appropriate antibiotic therapy was commenced and continued for three weeks with complete resolution of the parotid swelling and accompanying pyrexia. Subsequently the patient had three further attacks of staphyloccal septicaemia which on each occasion responded to courses of flucloxacillin and fusidic acid. No positive physical signs were elicited. Gynaecological examination was negative. Investigations to determine the source of the recurrent infection included plain X-rays and full contrast of studies of the gastrointestinal and genitourinary tract. No abnormalities were detected. Gallium-67 scan carried out

T.W. Evans, B.Sc., M.R.C.P.; R.S. Evely, M.B., Ch.B.; S.K. Morcos, F.R.C.S., F.R.C.R.

Correspondence: Dr T.W. Evans, Academic Division of Medicine, M Floor, Royal Hallamshire Hospital, Sheffield, S10 2JF.

Accepted: 3 May 1984 three months after presentation was normal. Shortly before the patient's death, plain X-ray of the pelvis showed bone erosion and widening of the symphysis pubis (Figure 1). A review of previous plain X-rays of this region indicated that these changes were progressive. Despite adequate antibiotic therapy the patient's condition rapidly deteriorated and she died. Post mortem examination revealed osteomyelitis of the symphysis pubis with an abscess cavity extending into the vulvae.

\section{Discussion}

The patient died as a result of septicaemia secondary to staphylococcal osteomyelitis of the symphysis pubis. Several features of the case are worthy of comment. Fewer than 50 cases of osteomyelitis of the symphysis pubis have been reported and in the majority of these immunological disorder, pelvic surgery, trauma or drug abuse preceded the onset of symptoms. Pain and tenderness at the site of infection were the presenting complaints (McHenry et al., 1975). In this case there was no predisposing cause and neither pain nor tenderness at the site of infection were ever encountered.

Plain X-rays showing irregularity and widening of the symphysis pubis may represent normal variations and are also seen post partum, and in instability of the symphysis pubis (Keats, 1979). The significance of these changes become obvious only when they are progressive as in this case or when associated with positive clinical signs and symptoms.

Gallium-67 scanning is said to be particularly sensitive in the detection of acute osteomyelitis even in the presence of chronic non inflammatory bone disease. Technetium-99 methylene diphosphonate $\left({ }^{99} \mathrm{Tc}^{\mathrm{m}} \mathrm{MDP}\right)$ scan has a detection rate close to $90 \%$. Gallium is positive in a greater proportion of cases as it 


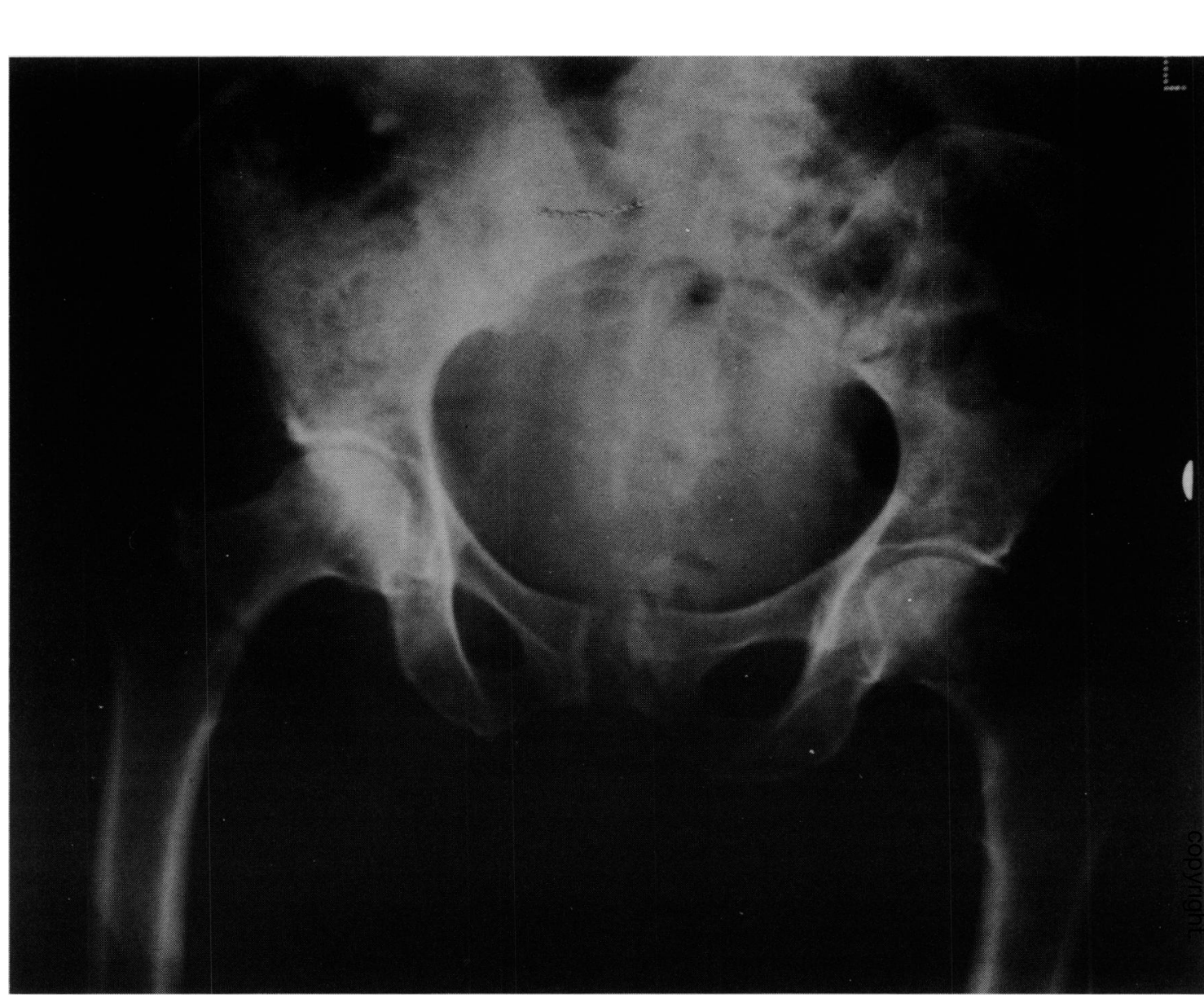

Figure 1 Plain X-ray of pelvis showing bone erosion and widening of the symphysis pubis.

is less dependent on blood flow for localization (Hoffer, 1982).

We report this case to demonstrate that osteomyelitis of the symphysis pubis may occur with no predisposing cause nor localized pain or tenderness. Furthermore the sensitivity of gallium-67 scintigraphy in detecting bone infection is questioned.

\section{References}

BUSTO R. DEL, QUINN, E.L., FISHER, E.I. \& MADHAVEN, T. (1982). Osteomyelitis of the pubis. Journal of the American Medical Association, 248, 1498.

HOFFER, P. (1982). Gallium and infection, Journal of Nuclear Medicine, 21, 484.

KEATS, T.E. (1979). An Atlas of Normal Roentgen Variants

\section{Acknowledgement}

Our thanks are due to Dr P. Howard, Reader in Medicine, University of Sheffield for his permission to report this case.

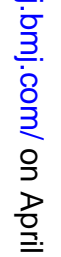

that May Simulate Disease. Second Ed. p. 196. Year book N Medical Publishers: Chicago, London.

McHENRY, M.C., ALFRIDI, R.J., WILDE, A.H. \& HAWK, W.A. 용 (1975). Hematogenous osteomyelitis: a changing disease. Cleveland Clinical Quarterly, 42, 124. 